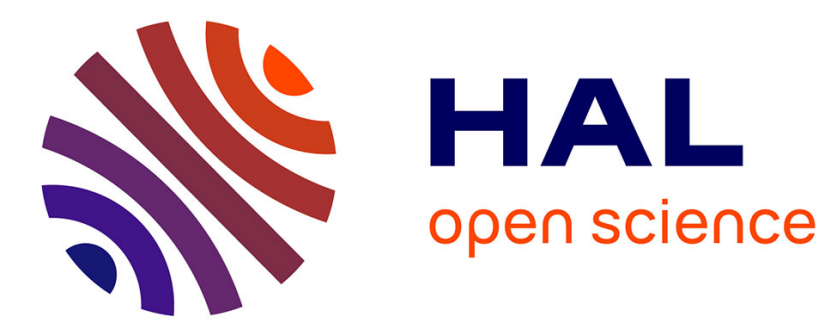

\title{
Current layer cascade in collisionless electron-magnetohydrodynamic reconnection and electron compressibility effects
}

\author{
Daniele del Sarto, Francesco Califano, Francesco Pegoraro
}

\section{- To cite this version:}

Daniele del Sarto, Francesco Califano, Francesco Pegoraro. Current layer cascade in collisionless electron-magnetohydrodynamic reconnection and electron compressibility effects. Physics of Plasmas, 2005, 12 (1), 10.1063/1.1829064 . hal-01796247

\section{HAL Id: hal-01796247 \\ https://hal.science/hal-01796247}

Submitted on 19 May 2018

HAL is a multi-disciplinary open access archive for the deposit and dissemination of scientific research documents, whether they are published or not. The documents may come from teaching and research institutions in France or abroad, or from public or private research centers.
L'archive ouverte pluridisciplinaire HAL, est destinée au dépôt et à la diffusion de documents scientifiques de niveau recherche, publiés ou non, émanant des établissements d'enseignement et de recherche français ou étrangers, des laboratoires publics ou privés. 


\title{
Current layer cascade in collisionless electron-magnetohydrodynamic reconnection and electron compressibility effects
}

\author{
D. Del Sarto, F. Califano, and F. Pegoraro ${ }^{\text {a) }}$ \\ Dipartimento di Fisica and INFM, Universita di Pisa, 56100 Pisa, Italy
}

(Received 31 August 2004; accepted 8 October 2004; published online 16 December 2004)

\begin{abstract}
The nonlinear phase of a magnetic field line reconnection instability in a collisionless two-dimensional cold plasma is investigated in the Hall dominated regime, described by the electron-magnetohydrodynamic equations, which corresponds to the frequency range of whistler waves. It is found that the regular pattern of current density layers that forms in the initial nonlinear phase of the reconnection instability is destroyed by the onset of a Kelvin-Helmholtz-type instability and the formation of current jets that develop into vortex rings. These processes can be interpreted in terms of a Hasegawa-Mima-type regime inside the magnetic island and lead to the creation of magnetic vortices. It is shown that electron compressibility, which is related to charge separation, tends to stabilize these processes. (C) 2005 American Institute of Physics.
\end{abstract}

[DOI: 10.1063/1.1829064]

\section{INTRODUCTION}

Understanding the mechanisms that control the time evolution of current layers in high conductivity plasmas and that determine their spatial distribution, and shape represents a basic step of all investigations of the nonlinear development magnetic field line reconnection processes, both in the magnetohydrodynamic (MHD) frequency range and in the higher frequency whistler range of electronmagnetohydrodynamics (EMHD) (see Ref. 1). In dissipationless (fluid) plasma regimes, where the effects of electric resistivity can be disregarded, magnetic reconnection is made possible by the current limitation due to the electron inertia terms in the generalized Ohm's law. In such Hamiltonian regimes the formation of narrow current structures was shown (see, e.g., Refs. 2-4 and references therein) to be an intrinsic consequence of the conservation of generalized topological invariants of the magnetic field configuration. These invariants are generalizations of the magnetic linking (connection) between plasma elements that is preserved in ideal MHD. In EMHD this linking involves the electron fluid elements since ions are assumed to be at rest. These topological invariants control the transformation of magnetic energy into plasma energy and, in EMHD, its redistribution between different field components.

In the present paper we investigate the evolution of the current layers that are formed in the nonlinear development of magnetic reconnection in the whistler frequency range by employing the EMHD equations. Within this simplified framework, electrons are treated as a cold electron fluid and electron inertia allows the magnetic field lines to decouple from the plasma motion. In this description quasineutrality is assumed and kinetic as well as dissipative effects are disregarded.

The nonlinear phase of collisionless magnetic reconnection in the whistler frequency range in a two-dimensional

\footnotetext{
${ }^{a)}$ Electronic mail: pegoraro@df.unipi.it
}

(2D) neutral line magnetic configuration, with a strong guide field in the direction perpendicular to the reconnection plane, shows the formation of a characteristic current pattern inside the magnetic island which consists of a current bar along the null line of the equilibrium magnetic field (see Ref. 5). Here we show that in the later nonlinear phase this current configuration becomes unstable toward a Kelvin-Helmholtztype (KH-type) instability which disrupts the regular structure of the current layer and leads to the formation of magnetic vortices inside the magnetic island. The onset of this secondary instability represents a feature that 2D collisionless EMHD reconnection shares with collisionless 2D MHD reconnection (see Ref. 2) in the cold electron fluid limit. In both cases compressibility effects tend to oppose the onset of the $\mathrm{KH}$ instability.

In the approach adopted here, the possible onset of additional secondary instabilities, involving both the electron and the ion dynamics and leading to anomalous momentum transport between the two species and thus to anomalous resistivity (see Refs. 6 and 7) is not included.

\section{A. Reconnection in the EMHD frequency range}

The set of the EMHD equations was first derived in Ref. 1 in order to describe the quasineutral dynamics of a plasma in the whistler frequency range, by assuming fluid (cold) electrons moving in a neutralizing background of ions at rest in such a way that the quasineutrality condition can be satisfied. This model applies to quasineutral plasma phenomena that occur on a characteristic time scale shorter than the ion cyclotron period $\left(\omega \gg \Omega_{c i}\right)$ and on spatial scales smaller than the ion skin depth $\left(L \ll d_{i}\right.$ with $d_{i}=c / \omega_{p i}$ and $\omega_{p i}$ the ion plasma frequency). In this regime Ohm's law is dominated by the Hall term (see Ref. 8) and in the absence of dissipation and for massless electrons, the magnetic field is frozen into the electron fluid.

As in the MHD case, current inhomogeneities can lead to reconnection instabilities (see Refs. 5, 9, and 10) that 
modify the magnetic field topology. ${ }^{11}$ As mentioned above, in this cold dissipationless fluid description, magnetic field line reconnection is made possible by the effect of the finite electron inertia. Magnetic reconnection in the whistler frequency regime was studied experimentally in laboratory plasmas (see Ref. 7, the related papers in the same issue and the references therein). It was reconsidered in Ref. 12 and, in the context of the nonlinear behavior of the quasistatic magnetic field generated during the interaction of ultrashort, ultraintense laser pulses with plasmas, in Ref. 13. In space plasmas, proof of the occurrence of collisionless reconnection processes in the Hall regime was found in recent Geotail observations (see Ref. 14).

The original investigations of collisionless magnetic reconnection, first performed in the MHD frequency range by Coppi in Ref. 15 in the fluid limit, were mostly motivated by applications to space plasmas processes such as reconnection events occurring in Earth's magnetotail (see Ref. 16) where reconnection arises because of phase-space effects. Interest in collisionless reconnection has been growing considerably in recent years in the context of study of the nonlinear development, of the time scales, and of the saturation amplitudes of reconnection instabilities in the low collisionality, high temperature, magnetically confined plasmas of present day laboratory fusion experiments (see Refs. 3, 4, 17, and 18).

Here we will restrict ourselves to $2 \mathrm{D}$ plasma fluid regimes where kinetic (phase-space) effects are disregarded and a strong uniform external magnetic field is imposed, and focus our attention on the nonlinear development of EMHD collisionless reconnection and on the onset of secondary "fluid" instabilities that affect the cascade of the current density distribution towards small spatial scales. Small scale secondary instabilities resulting from the current dynamics have been recently discussed in Ref. 19, where reconnection in a low $\beta$ MHD regime was considered. Our analysis is related to the one presented in Ref. 2 where, however, the main interest was on the development of collisionless fluid reconnection in the MHD frequency range and the EMHD results were only briefly mentioned.

\section{B. EMHD vortices and $\mathrm{KH}$ instability}

Extensive work has been devoted in the literature to the investigation of the $\mathrm{KH}$ instability in magnetized plasmas within the MHD and EMHD frameworks, mostly because of the importance that vortex dynamics plays in a variety of nonlinear processes in magnetized plasmas and, in particular, in the development of turbulence. In Ref. 20 the KH instability was studied in a 2D Hall dominated MHD theory and shown to generate short wavelength turbulence, while the role of the electron Kelvin-Helmholtz instability for a current layer narrower than the electron collisionless skin depth $d_{e}=c / \omega_{p e}$ was also pointed out in Ref. 21. Vortices, localized exponentially in a region of the order of $d_{e}$, were studied in Ref. 22 in the framework of the 2D and 3D EMHD equations. In the high frequency range of interest for the interaction of ultraintense laser pulses with a plasma, magnetic vortices in the electron fluid were shown ${ }^{23}$ to be unstable to the
$\mathrm{KH}$ instability when the vortex distance is shorter than vortex screening radius which is of order $d_{e}$. In Ref. 24, the onset of fractal turbulent structures in the case of the 2D KH instability in an incompressible fluid plasma was related to the presence of Lagrangian invariants (see Sec. II B later in the present paper). The interplay between the KH instability and reconnection instabilities in plasma configurations with strong sheared flows was investigated analytically and numerically in a resistive-viscous plasma in Ref. 25 and in the Hall-MHD limit in Ref. 26. A study both of the evolution of a current-vortex sheet and a plane fluid jet in a neutral magnetic sheet was performed in Ref. 27. The twisting of the "frozen" magnetic lines due to the compression of the fluid as a consequence of the $\mathrm{KH}$ has proved to enhance the growth rate of the resistive reconnection instabilities by increasing the current gradients; in Ref. 28 this mechanism is argued to play a role in the reconnection phenomena in the solar corona. The relationship between $\mathrm{KH}$ and reconnection instabilities was indeed examined for various astrophysical environments: in Refs. 29 and 30 reconnection was shown to occur as consequence of the fluid instability; in Ref. $31 \mathrm{KH}$ vortex motion has been indicated as a possible cause for a mass transport into the terrestrial magnetotail, responsible for the observed high density and low temperature filaments in the magnetosphere.

In more recent studies (see Ref. 32) performed in the 2D incompressible MHD model it was also suggested that in the EMHD collisionless limit the $\mathrm{KH}$ and the tearing mode are both unstable but not interacting. In the present paper we show that KH-type instabilities occur in this regime as a consequence of the field configuration induced by the presence of the topological constraints during this tearingreconnection process; because of the analogous dynamical behavior characterized by the same topological invariants, this phenomenon is observed also in a collisionless 2D "cold-electron" MHD framework. Finally we recall that in the literature it is argued (see Refs. 20 and 33) that finite ion gyroradius effects, associated with an anisotropic ion stress tensor, have a stabilizing effect on the KH MHD instability. This is reminiscent of the recent results presented in Ref. 2 in the case of 2D collisionless MHD reconnection, where the onset of the secondary KH instability in the nonlinear reconnection phase appears to be stabilized by the introduction of the so-called ion-sound gyroradius terms. These terms are related, through the perturbed electron stress tensor (see Refs. 4 and 34), to the electron compressibility along the magnetic field lines. This is in agreement with the wellknown hydrodynamic result that fluid compressibility stabilizes the KH mode (see, e.g., Ref. 35, p. 74). Thus, within the EMHD framework, it is interesting to investigate whether electron compressibility, which modifies the EMHD equations (see Refs. 36 and 37) by relaxing the quasineutrality requirement, can effectively modify the development of the $\mathrm{KH}$ instability. Our numerical results confirm that this is the case, in agreement with the results recently presented in Ref. 38 which show that fluid (i.e., ion) compressibility stabilizes the MHD KH disturbances. 


\section{EMHD EQUATIONS, GENERALIZED MAGNETIC CONNECTIONS, AND ELECTRON COMPRESSIBILITY}

The equations describing the plasma dynamics in the collisionless EMHD framework can be combined (assuming electron flow incompressibility $\boldsymbol{\nabla} \cdot \mathbf{u}_{e}=0$ so as to maintain quasineutrality in a homogeneous plasma) into a single expression for the temporal evolution of the magnetic field $\mathbf{B}$ (see Ref. 1),

$$
\partial_{t} \mathbf{\Omega}=-\alpha \boldsymbol{\nabla} \times[(\boldsymbol{\nabla} \times \mathbf{B}) \times \mathbf{\Omega}],
$$

where $\boldsymbol{\Omega}=\mathbf{B}-d_{e}^{2} \nabla^{2} \mathbf{B}$ is the generalized vorticity, $\alpha$ $=c / 4 \pi n_{e} e$ is the Hall coefficient, and the electron velocity $\mathbf{u}_{e}$ and magnetic field are connected by Ampère's law $\mathbf{u}_{e}$ $=-\alpha \nabla \times \mathbf{B}$. From Eq. (1) we see that the generalized vorticity $\Omega$ is frozen in the electron flow, i.e., if two electron fluid element are initially linked by an $\Omega$-field line, they remain linked at all times.

In a $2 \mathrm{D}$ configuration we write the magnetic field $\mathbf{B}$ $=\mathbf{B}(x, y, t)$ in terms of two scalar functions $\psi(x, y, t)$, $b(x, y, t)$ as

$$
\mathbf{B}=\mathbf{b}_{\perp}+b \mathbf{e}_{z}=\boldsymbol{\nabla} \psi \times \mathbf{e}_{z}+b \mathbf{e}_{z},
$$

so that Eq. (1) take the form (see Refs. 2 and 5)

$$
\begin{aligned}
& \partial_{t} F+[b, F]=0, \\
& \partial_{t} W+[b, W]=[\psi, F] / d_{e}^{2},
\end{aligned}
$$

with $F=\psi-d_{e}^{2} \nabla^{2} \psi, W=b-d_{e}^{2} \nabla^{2} b$, and $[A, B]=\mathbf{e}_{z} \cdot \boldsymbol{\nabla} A \times \boldsymbol{\nabla} B$. Dimensionless variables are used based on the characteristic equilibrium scale length $L$ and on the corresponding whistler time $\tau_{w}=\left(d_{e}^{2} \Omega_{c e}\right)^{-1}$, where $d_{e}=c /\left(L \omega_{p e}\right)$ is the normalized electron skin depth, $\Omega_{c e}=e B_{\infty}^{0} /\left(m_{e} c\right)$, where $B_{\infty}^{0}$ is a reference value of the magnetic field. We recall that whistlers waves play in the EMHD regime a role analogous to that of Alfvén's waves in the MHD regime. However, on the contrary to Alfvén waves, their dispersion relation, which is given in normalized units by

$$
\omega_{w}(\mathbf{k})=\alpha \frac{\left(\mathbf{B}_{0} \cdot \mathbf{k}\right) k}{1+k^{2} d_{e}^{2}},
$$

is dispersive.

We also recall that, as mentioned in Sec. I A, the assumption of immobile ions restricts us to time scales shorter than the ion cyclotron time. Thus it can be expected that the ion dynamics will start to affect the EMHD nonlinear behavior after a time (in normalized units) of order $d_{e}^{2}\left(m_{i} / Z_{i} m_{e}\right)$, where $m_{i}$ and $Z_{i}$ are the ion mass and charge number. In order to focus explicitly on the phenomena governed by the dynamics of the electrons, in the present analysis we disregard this effect and take formally the limit $m_{i} / Z_{i} \rightarrow \infty$.

In the special case where $F \equiv 0$, the system of Eqs. (3) and (4) reduces to the Hasegawa-Mima Equation: ${ }^{40}$

$$
\partial_{t} W+[b, W]=0,
$$

which has been studied recently, e.g., in the context of the magnetic field generation and dynamics by an ultrashort and ultraintense laser pulse propagating in an underdense plasma. $^{23}$
This incompressible model applies when $\omega<\Omega_{c e} \ll \omega_{p e}$. We recall that in this case the electron current density in the $x-y$ plane is proportional, through constant terms, to the electron velocity in this plane so that the magnetic field components $b$ plays the role of the stream function (or equivalently of the electrostatic potential) $\varphi$ used for the MHD case in Refs. 2-4. For this reason in the following we denote as fluid regimes, as opposed to "magnetic" regimes, those regimes where the effect of $b$ dominates over that of $\psi$, as is the case, e.g., with Eq. (6), even if in both cases the energies involved are magnetic (see Sec. II B below).

Corrections due to electron compressibility in a plasma where $\Omega_{c e} \gtrsim \omega_{p e}$ were studied in Refs. 36 and 37. In the 2D configuration adopted here, compressibility acts on the electron motion in the $x-y$ plane only and leaves Eqs. (3) and (4) substantially unchanged, but for a redefinition of $W$ in Eq. (4),

$$
W \rightarrow W^{*}=b-\lambda_{e}^{2} \nabla^{2} b, \quad \text { with } \lambda_{e}^{2}=d_{e}^{2}\left(1+\Omega_{c e}^{2} / \omega_{p e}^{2}\right) .
$$

We notice that since the ions are static, electron compressibility in EMHD corresponds to a violation of quasineutrality, differently from what happens in 2D MHD regimes where plasma quasineutrality can always be preserved even if compressibility effects are taken in account.

\section{A. Linear growth rate of the 2D EMHD reconnection instabilities}

The linear phase and the growth rate $\gamma$ of the EMHD reconnection instability are given in Refs. 10, 37, and 39 where Eqs. (3) and (4) are linearized in the form

$$
\begin{aligned}
& \psi(x, y, t)=\psi_{0}(x)+\psi_{1}(x) \exp (i k y+\gamma t), \\
& b(x, y, t)=b_{0}+b_{1}(x) \exp (i k y+\gamma t),
\end{aligned}
$$

where $b_{0}$ is a constant and $\psi_{0}(x)$ describes an equilibrium configuration with a null line. The general dispersion relation in the small $d_{e}$ limit and over the full range of values $\Delta^{\prime} d_{e}$ (here $\Delta^{\prime}$ is the standard jump parameter of reconnection instabilities) was obtained for an incompressible electron fluid in Ref. 39 with a boundary layer approach. In Ref. 37 compressibility effects were shown to reduce the growth rate of the EMHD reconnection instability.

\section{B. Hamiltonian structure of the 2D EMHD equations and Lagrangian invariants}

As is well known Eqs. (3) and (4) are Hamiltonian (see Ref. 34) with energy functional

$$
\mathcal{H}=\frac{1}{2} \int\left(|\nabla \psi|^{2}+|b|^{2}+d_{e}^{2}\left|\nabla^{2} \psi\right|^{2}+\lambda_{e}^{2}|\nabla b|^{2}\right) d x d y,
$$

given by the sum of the magnetic and of the kinetic electron energies. In Eq. (3) the $z$ component of magnetic field $b$ acts as a "stream function" of the velocity field that advects the generalized flux function $F$ as a Lagrangian invariant. The invariance of $F=\psi-d_{e}^{2} \nabla^{2} \psi=\psi+d_{e}^{2} J_{z}$ is related to the conservation of the canonical electron momentum along $z$ and represents the $2 \mathrm{D}$ form of the generalized linking condition discussed above in terms of the generalized vorticity field $\boldsymbol{\Omega}$. 
For configurations with scale lengths much larger than $d_{e}$, $F \rightarrow \psi$ and the generalized connections reduce to the standard magnetic connections in the $x-y$ plane determined by the isocurves of $\psi$. On the contrary, $W$ in Eq. (4) is not a Lagrangian invariant, while its line integral along a field line of the generalized magnetic flux function $F$ is a Lagrangian invariant. These invariance properties can be expressed in terms of two infinite sets (see Refs. 10 and 34) of global topological invariants (Casimirs) defined as $C_{1}=\int d x d y \mathcal{F}(F)$ and $C_{2}=\int d x d y W \mathcal{G}(F)$ with $\mathcal{F}$ and $\mathcal{G}$ arbitrary smooth functions.

As already pointed out in Ref. 2, the algebraic structure and the invariance properties of Eqs. (3) and (4) coincide with those of the two-fluid 2D MHD model discussed in Refs. 3, 4, and 34 in the limit of cold electrons (i.e., when the so-called ion-sound gyroradius $\rho_{s}$ vanishes) as can be seen, e.g., by comparing Eqs. (3) and (4) with Eqs. (1) of Ref. 2 and by substituting the electrostatic potential $\varphi$ for the $z$-component $b$ of the magnetic field. However, the system of equations is different as the time derivative in the second of Eqs. (1) of Ref. 2 acts on $\nabla^{2} \varphi$, while in Eq. (4) it acts on $W=b-d_{e}^{2} \nabla^{2} b$. This difference is reflected in the energy functionals, as the term corresponding to $|b|^{2}$ in Eq. (10) (which in EMHD allows for the magnetic energy redistribution between the components of the magnetic field in the $x-y$ plane and the component along $z$ ) is absent in the two-fluid 2D MHD model. In Refs. 2 and 4 it was shown that the nonlinear development of the reconnection instability is characterized by the mixing of the Lagrangian invariants in the $x-y$ plane and by the formation of increasingly small spatial scales in the current density and vorticity distribution, accompanied by the transfer of magnetic energy towards these small scales. In the cold-electron limit this mixing becomes turbulent due to the onset of a secondary KH instability, as mentioned in the Introduction. As the electron temperature is increased, the effect of the parallel electron compressibility, which enters through the ion-sound gyroradius $\rho_{s}$, turns the mixing of the Lagrangian invariants from turbulent to laminar by suppressing the onset of the KH instability. Here we show that the same mechanism of turbulent mixing occurs in the nonlinear development of the reconnection instability in the incompressible EMHD case. The correspondence between the behaviors of these two regimes is indeed consistent with the "small-scale" equivalence of the two dynamical systems. When spatial scales much smaller than $d_{e}$ are formed, $\partial_{t} b \ll d_{e}^{2} \partial_{t}\left(\nabla^{2} b\right)$ so that, at these spatial scales, Eq. (4) [or Eq. (7)] becomes equivalent to the second of Eqs. (1) of Ref. 2 after identifying $d_{e}^{2} \nabla^{2} b \leftrightarrow U$, where $U$ is the vorticity of the fluid MHD plasma motion in the $x-y$ plane, and the two regimes share the same Hamiltonian structure.

\section{NUMERICAL RESULTS}

In order to investigate the fully nonlinear phase of the EMHD reconnection instability we have integrated Eqs. (3) and (4) numerically by means of the 2D fluid code described in Ref. 2 for values of $d_{e}$ ranging from $0.06 \mathrm{~L}$ to $1 \mathrm{~L}$. Subsequently, we have investigated the effect of electron compressibility by letting $\lambda_{e}$ vary at fixed $d_{e}$. The numerical com-

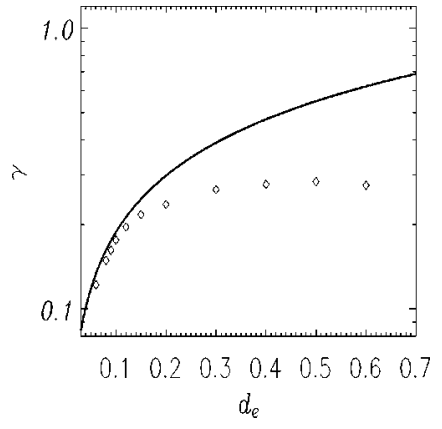

FIG. 1. Scaling of the growth rate of the reconnection instability with $d_{e}$. The solid line represents the analytical prediction for the $d_{e} \Delta^{\prime}>1$ ordering; the diamonds represent the numerically obtained values.

putations are initialized in a simulation box with $L_{x}=2 L_{y}$ $=4 \pi L$ on a $1024 \times 512$ mesh, with an equilibrium configuration given by $\psi_{0}(x)=-L /\left[2 \cosh ^{2}(x / L)\right]$ and $b_{0}=$ const. For $d_{e}=0.12 L, 0.3 L$ we have run additional simulations with higher resolution, $2048 \times 512$. The stiffness of the dispersion relation of whistler waves [Eq. (5)] imposes strong restrictions on the use of finer mesh and smaller values of the electron skin depth. In Fig. 1 we plot the numerically obtained values of the linear growth rates of the most unstable mode $(k=1)$ for $0.06 L<d_{e}<1.0 L$, against the analytical values (solid line) derived in Ref. 39 with a boundary layer theory valid for small values of $d_{e}$. In this limit, the scaling, predicted in Eq. (37) of Ref. 39 for large value of the socalled tearing instability parameter $\Delta^{\prime}$, is well verified (for our choice of the equilibrium magnetic field profile, the $k$ $=1$ mode has $\Delta^{\prime} \simeq 14.3$ ).

\section{A. Current layers cascade and fluid instabilities}

Here we present the results of a simulation with $d_{e}$ $=0.3$; the corresponding linear instability growth rate is $\gamma$ $=0.55$. The magnetic flux function $\psi$ displays a smooth structure with a finite size magnetic island and reconnected field lines, as shown by the solid lines in Fig. 2 at $t=147,153$, 155 , and 157 . On the contrary, the contour lines of the Lagrangian invariant $F$ do not reconnect, as shown by the shaded contours in Fig. 2, and are advected along the hyperbolic cell pattern of the stream function $b$ (shown in Fig. 3(a) at $t=147)$. Initially, this advection pattern bends the $F$ $=$ const lines toward the $X$ point of the magnetic island so that a bar-shaped current layer (current jets) is formed along the equilibrium null line, as Figs. 4(a) and 4(b). Such a bar structure is also apparent in the $F$ contours [see, e.g., 2(a) and 2(b). Subsequently, this pattern causes the current jets, coming from two adjacent $X$ points, to move in the $y$ direction toward the $O$ point. Since $F$ cannot reconnect when the jets meet [Figs. 2(c) and 4(c)] they are deflected in the $x$ direction towards the island separatrix (Figs. 2(d) and 4(d)). The onset of secondary (KH)-type instabilities prevents the streams of currents from eventually filling the whole [Figs. 5(a) and 5(b)] with a "fractal" sequence of secondary layers, roughly perpendicular to each other, that would develop because of the deflections that occur when two secondary jets collide either among them or with the island separatrix. In- 

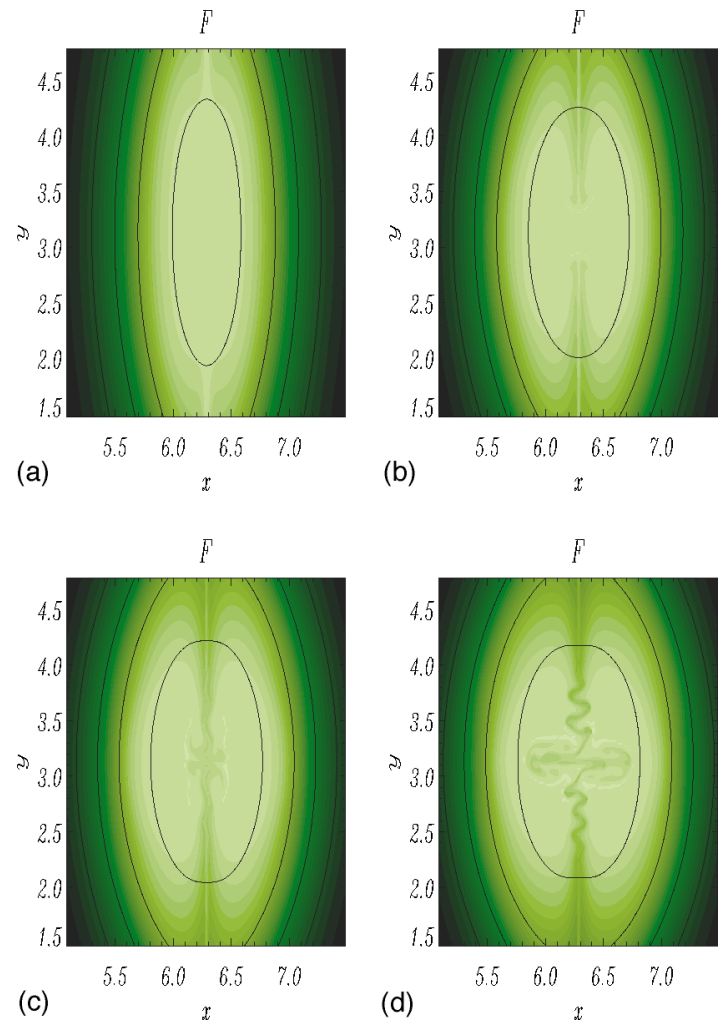

FIG. 2. Shaded isocontours of the Lagrangian invariant $F$ at $t=147,153$, 155 , and 157 for $d_{e}=\lambda_{e}=0.3$. Superimposed (solid lines) are the isocontours of the magnetic flux function $\psi$.

deed, this model evolution of the current layers, in a cascade resembling the iteration algorithm of a Peano-Hilbert fractal structure (see Ref. 41), cannot be followed after the second iteration because the $\mathrm{KH}$ fluid instability starts to develop even before the first deflection at the $O$ point. The time at which this instability modifies the pattern of $J_{z}$ depends on the strength and width of the current bar along the neutral line, i.e., on the magnitude of the parameter $d_{e}$. When measured on the time scale that characterizes the advection of $F$, we find that larger values of $d_{e}$ make the KH instability develop later (cf. Figs. 6(a) and 4(d)). This behavior can be interpreted by recalling that the width of the reconnection zone, and thus of both the current and the vorticity layers,
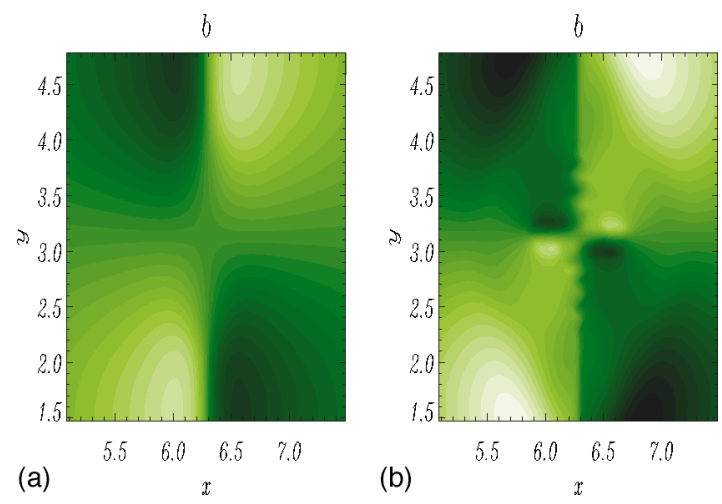

FIG. 3. Pattern of the stream function $b$ near the $O$ point of the magnetic island at $t=147$ and $157\left(d_{e}=\lambda_{e}=0.3\right)$.
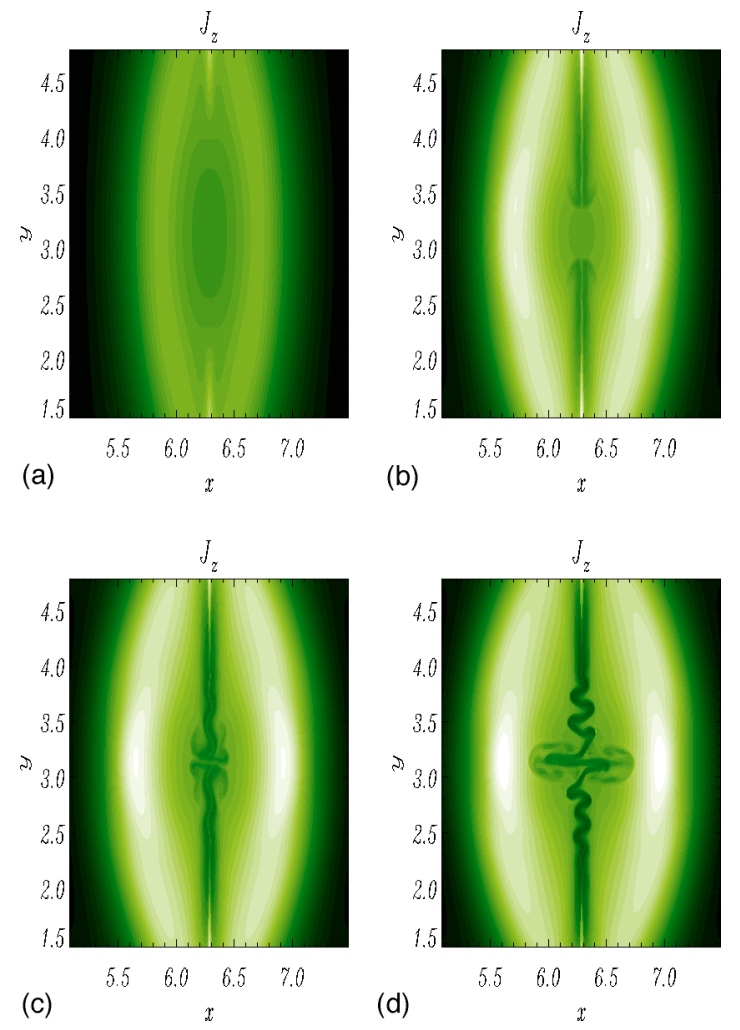

FIG. 4. Shaded isocontours of the electric current $J_{z}$ at $t=147,153,155$, and $157\left(d_{e}=\lambda_{e}=0.3\right)$.

grows with $d_{e}$ and that a broader layer means smoother gradients and thus a lower $\mathrm{KH}$ growth rate, even though the peaks of the velocity near the neutral line can rise when $d_{e}$ is increased.

The observed instability can be referred to more properly as a kind of sinuous Bickley's jet instability (see, e.g., Ref. 35). As clearly visible from the figures, this fluid instability combines with the development of pairs of vortices due to the rolling up of the vortex sheets (see, e.g., Ref. 42) at the edge of the current bars where it produces the characteristic "mushroom-patterns" seen, e.g., in Figs. 2(b) and 4(b) and at different regimes, in Figs. 6(a) and 6(b). The development of these vortex structures can be interpreted as a local change of the plasma evolution along the current jets inside the magnetic island from a regime where both components of the magnetic field, represented by the two functions $b$ and $F$, respectively, determine the plasma dynamics to a regime where the plasma dynamics near the current jets is dominated by the field $b$, i.e., by the component of the magnetic field out of the $x-y$ plane [and its related "vorticity" shown in Figs. 7(a) and 7(b) ]. In the limit where the contribution of the term $[\psi, F] / d_{e}^{2}$ is neglected in Eq. (4), this regime is governed by the Hasegawa-Mima-type Eq. (6). This equation describes the dynamics of fluidlike vortices characterized by a screening length given by $d_{e}$. On spatial scales smaller than $d_{e}\left[\partial_{t} b<d_{e}^{2} \partial_{t}\left(\nabla^{2} b\right)\right]$ these vortices reduce to hydrodynamic vortices with a logarithmic stream function in the case of a pointlike vorticity distribution. The interaction between such screened vortices and the stability of the resulting structures has been studied in Ref. 23. 


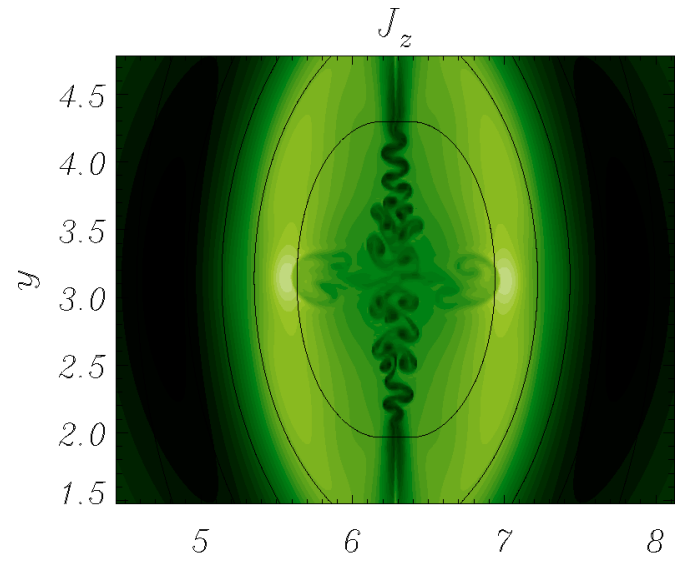

(a)

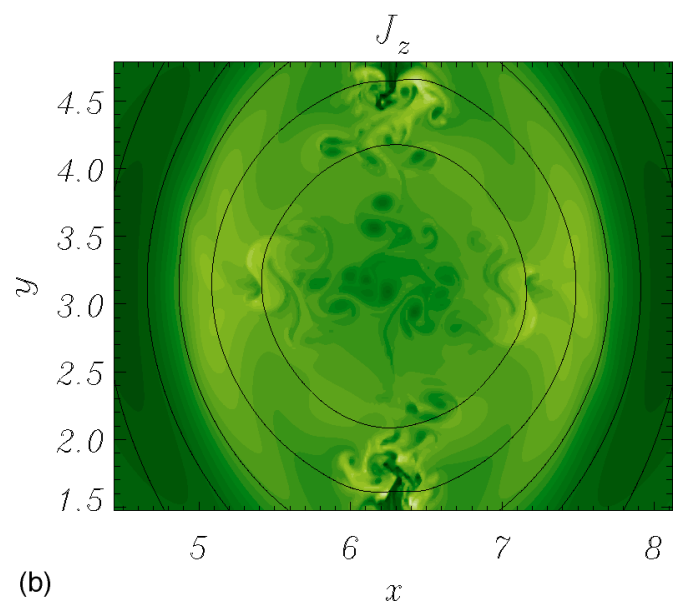

FIG. 5. Shaded isocontours of $J_{z}$ at $t=159$ and 167 with the superimposed contours of the magnetic island $\left(d_{e}=\lambda_{e}=0.3\right)$.

\section{B. Stabilizing effects of electron compressibility and of magnetic effects}

The effect of the electron compressibility on the formation of vortices, and on their subsequent interaction leading to the onset of a turbulent regime, is shown in Figs. 6(a) and 6(b) for $d_{e}=0.3 L$ and $\lambda_{e}=0.67 L$ [see Eq. (7)]. The stabilizing effect of compressibility is evident in Fig. 6(c) where the first collision between the current jets occurs before these layers are distorted by the fluid (KH)-type modes.

In order to verify this effect more directly, we have initialized numerical simulations with a simplified magnetic configuration constructed so as to model the region along the null line of the shear magnetic field. This model configuration is characterized by a narrow layer of the current $J_{z}$, roughly similar to the one observed inside the island not far from the $O$ point in Fig. 2(b), and by a fluid velocity $\mathbf{u}$ $=u_{0} \mathbf{e}_{y}$ with $u_{0} \approx 0.085$ (in $L / \tau_{w}$ units), peaked in a layer of thickness $\delta \simeq d_{e}$. The numerical parameters of these runs are $L_{x}=2 \pi L=L_{y} ; N_{x}=1024=N_{y}, d_{e}=0.3 L$, and $\lambda_{e}$ equal to $d_{e}$ and $d_{e} \sqrt{5}$. For $\lambda_{e}=d_{e}$ the KH instability develops with a growth rate of the order of the whistler time. On the contrary, in the compressible case $\left(\lambda_{e}=0.67 L\right)$ no appreciable changes in the initial velocity flow have been observed over a time scale of
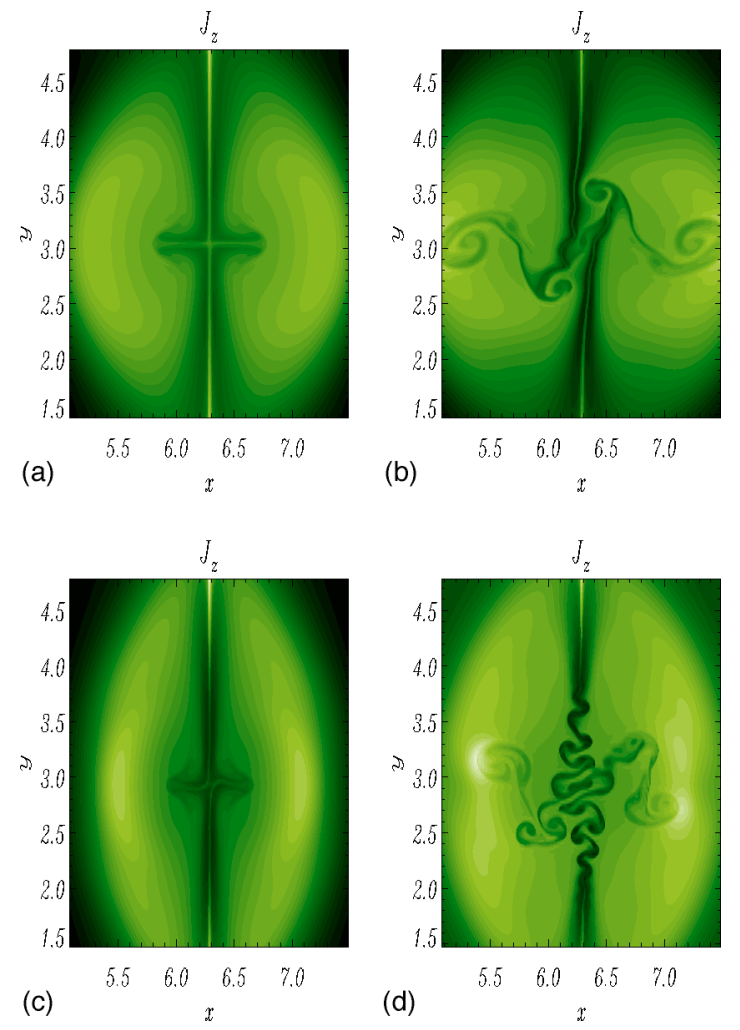

FIG. 6. Shaded isocontours of $J_{z}$ at $t=87$ (a) and $t=90$ (b) in the incompressible case with $d_{e}=\lambda_{e}=0.6$ and at $t=262$ (c) and $t=270$ (d) in the compressible case with $d_{e}=0.3$ and $\lambda_{e}=0.67$. In both regimes the development of the KH-l instability is delayed [see Fig. 4(d)].

nearly 20 whistler times. These numerical results are in agreement with those presented in Ref. 38 regarding a MHD regime with a weak magnetic field.

\section{Conservations and energy exchange}

The numerical accuracy of the simulations presented above is supported by the conservation of the invariant $\mathrm{Ca}$ simirs (defined in Sec. II B), not shown here, and of the total energy shown in Fig. 8(a) for the incompressible $d_{e}=0.3 \mathrm{~L}$ case, and in Fig. 9(a) for $\lambda_{e}=\sqrt{5} d_{e}=0.67 L$. Note that in this section, the energy related to $b$ is shown with its initial constant value subtracted. The conservation of the Casimirs (we
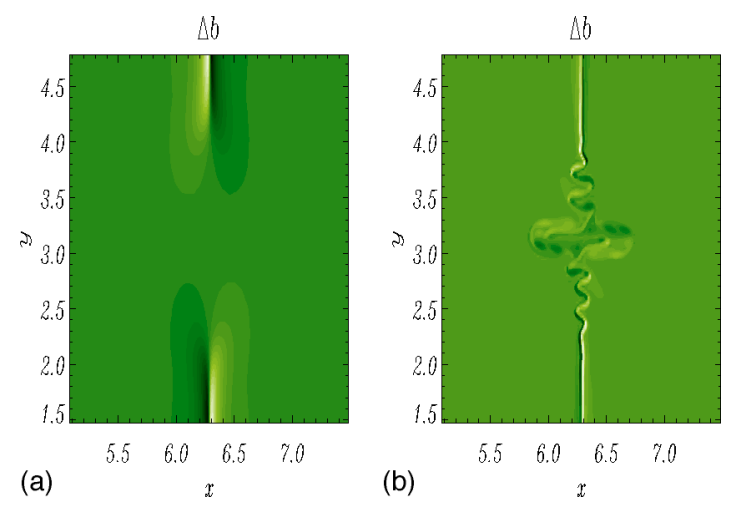

FIG. 7. Shaded isocontours of the $b$-related vorticity $\Delta b$, at $t=147$ and 157 $\left(d_{e}=\lambda_{e}=0.3\right)$. 


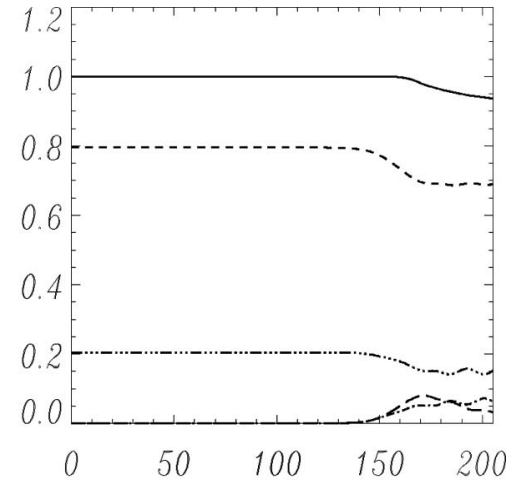

(a)

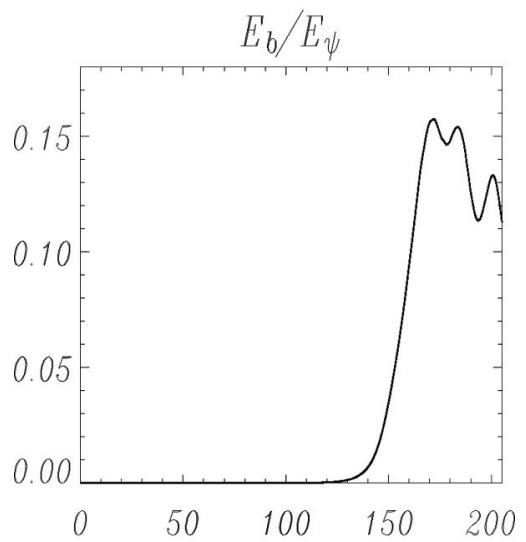

(b)

$\tau_{w}$

FIG. 8. Energy balance vs time (a) and oscillatory energy exchange between magnetic and fluid energies (b) for the incompressible case with $d_{e}=\lambda_{e}$ $=0.3$. In (a) symbols are as follows: continuous line $\leftrightarrow$ total energy, dashed line $\leftrightarrow|\nabla \psi|^{2}=\left|\mathbf{b}_{\perp}\right|^{2}$, three-dot-dashed line $\leftrightarrow d_{e}^{2}\left|\mathbf{J}_{z}\right|^{2}$, long-dashed line $\leftrightarrow|b|^{2}$ $=\left|\mathbf{b}_{z}\right|^{2}$, dot-dashed line $\leftrightarrow d_{e}^{2}\left|\mathbf{J}_{\perp}\right|^{2}$.

have considered the integrals over the $x$-y plane of $F, F^{2}, F^{3}$, and $F^{4}$ ) and of the total energy are optimal (less than $1 \%$ of loss) until the saturation phase is reached, which in the incompressible case with $d_{e}=0.3 L$ corresponding approximately to the time $t=150$ and to $t=250$ for $\lambda_{e}=\sqrt{5} d_{e}$ $=0.67 \mathrm{~L}$ (see Figs. 8(a) and 9(a) for what concerns the energy balance). At later times the formation of ever decreasing spatial scales breaks the Hamiltonian properties of the system numerically. Nevertheless, the total energy, which turns out to be the worst conserved quantity, remains at the end of our simulation within a $5 \%$ of its initial value in the $d_{e}=0.3$ case and within $10 \%$ of its initial value in the $\lambda_{e}=\sqrt{5} d_{e}=0.67$. A similar behavior has been observed for the other values of $d_{e}$ and $\lambda_{e}$.

In Figs. 8(a) and 9(a) it is shown that the difference between the magnetic energy of the initial and of the final configurations is transferred towards the increasingly small spatial scales of the current distributions $\nabla^{2} \psi$ along $z$ and $\nabla b$ in the $x-y$ plane. We remark that for the parameters of the simulations the "kinetic energy" $\left(d_{e}^{2}\left|\nabla^{2} \psi\right|^{2}=d_{e}^{2}\left|\mathbf{J}_{z}\right|^{2}\right)$ due to the equilibrium current may represent an important source of energy. In the nonlinear saturated phase, after numerous vortices have been generated, the coupling of magnetic and fluid energies manifests itself as an oscillatory exchange of energy
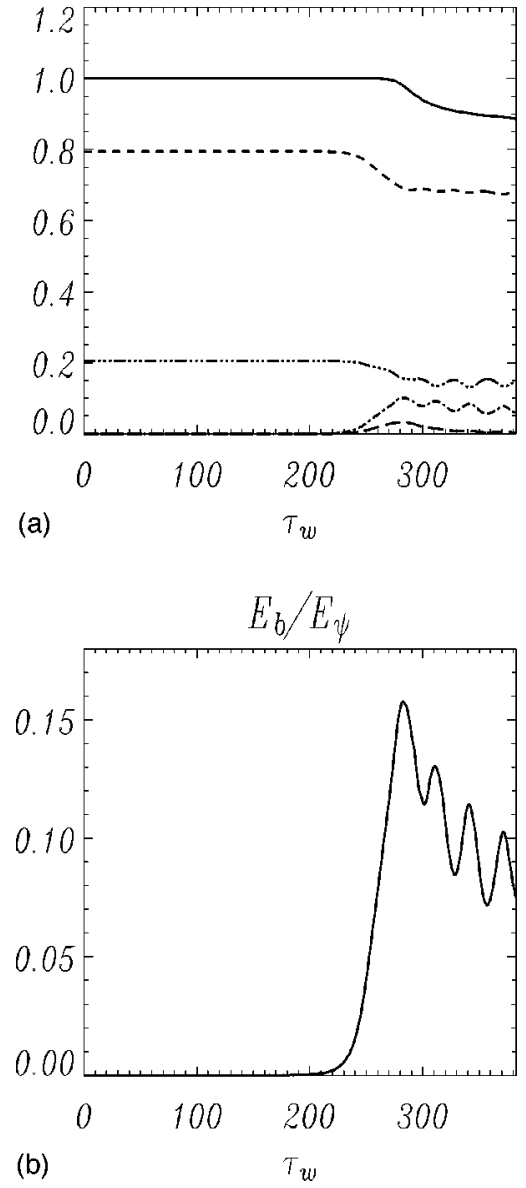

FIG. 9. Energy balance vs time (a) and oscillatory energy exchange between magnetic and fluid energies (b) for $\lambda_{e}=\sqrt{5} d_{e}=0.67$. Same symbols as in Fig. 8 .

between the $\psi$-dependent terms on one side (the shear magnetic energy $|\boldsymbol{\nabla} \psi|^{2}=\left|\mathbf{b}_{\perp}\right|^{2}$ and the " $z$ " kinetic energy $d_{e}^{2}\left|\nabla^{2} \psi\right|^{2}=d_{e}^{2}\left|\mathbf{J}_{z}\right|^{2}$ ) and the $b$-dependent terms on the other (the $z$ magnetic energy $\left(|b|^{2}\right)$ and " $x-y$ " kinetic energy $\left(d_{e}^{2}|\boldsymbol{\nabla} b|^{2}=d_{e}^{2}\left|\mathbf{J}_{\perp}\right|^{2}\right)$. As already mentioned in Ref. 2 this process is accompanied by oscillations of the island shape and is analogous to that described in Ref. 43. It is also worth noticing that the two components of the total energy, the fluid energies $E_{b}=|b|^{2}+d_{e}^{2}\left|\mathbf{J}_{\perp}\right|^{2}$ and the magnetic energies $E_{\psi}$ $=\left|\mathbf{b}_{\perp}\right|^{2}+d_{e}^{2}\left|\mathbf{J}_{z}\right|^{2}$, exhibit the same behavior observed in Ref. 44 , the ratio $E_{b} / E_{\psi}$ being brought from its initial value (here appearing as "zero") to a constant value smaller than one through the nonlinear exchange, almost independently of the magnitude of $\lambda_{e}$ [see Figs. 8(b) and 9(b)]. The frequency of these energy oscillations is influenced by $\lambda_{e}$ and decreases as $\lambda_{e}$ increases. As mentioned above, these energy oscillations are related to pulsations of the magnetic island. In Ref. 38 an analogous exchange between magnetic and fluid-kinetic energy observed in the Hall-MHD regime is motivated as due to the alternate saturation of secondary vortices and their disruption because of the magnetic reconnection.

Our simulations show that these nonlinear oscillations tend to damp. The decrease of their amplitude is accompanied by a progressive decrease of their frequency which is 
reminiscent of the results presented in Ref. 45 (and references therein) for the nonlinear dynamics of a driven system.

\section{CONCLUSIONS}

We have investigated the saturation process of the collisionless EMHD reconnection instability and the related mixing of the Lagrangian invariants. We have compared its nonlinear development to that analyzed in the cold-collisionless MHD regime in Ref. 2 . We have shown that mixing of the Lagrangian invariants is a phenomenon common to different types of 2D Hamiltonian reconnection.

We have also shown that in the EMHD regime, where the electrons are treated as a cold fluid and the ion are taken to be immobile, a secondary instability of the KelvinHelmoltz-type arises, similarly to the cold-electron limit of MHD reconnection discussed in Ref. 2. In these cold regimes the process of mixing of the Lagrangian invariant is turbulent. We have shown that inside the magnetic island, near the current jets, the plasma dynamics is of a "fluid type" (i.e., in the EMHD regime it is dominated by the effect of the magnetic field component $b$ out of the $x-y$ plane and is approximately described a Hasegawa-Mima equation).

We have analyzed the effect of electron compressibility, which in EMHD is related to charge separation. We have shown that the transition to the turbulent regime, due to the interaction of fluid vortices generated by the onset of a Kelvin-Helmoltz instability, is delayed by electron compressibility effects.

An oscillatory energy exchange between the magnetic and the "fluid" energy components is observed in the advanced saturated phase of the reconnection instability. This exchange is accompanied by pulsations of the magnetic island.

\section{ACKNOWLEDGMENTS}

Useful discussions with A. Mangeney and A. F. Rappazzo are gratefully acknowledged.

Part of this work was supported by the INFM Parallel Computing Initiative, by the Italian Ministry for University, and by Scientific Research PRIN 2002 funds.

\footnotetext{
${ }^{1}$ A. S. Kingsep, K. V. Chukbar, and V. V. Yan'kov, in Reviews of Plasma Physics, edited by B. Kadomtsev (Consultants Bureau, New York, 1990) Vol. 16, p. 243.

${ }^{2}$ D. del Sarto, F. Califano, and F. Pegoraro, Phys. Rev. Lett. 91, 235001 (2003).

${ }^{3}$ E. Cafaro, D. Grasso, F. Pegoraro, F. Porcelli, and A. Saluzzi, Phys. Rev. Lett. 80, 4430 (1998).

${ }^{4}$ D. Grasso, F. Califano, F. Pegoraro, and F. Porcelli, Phys. Rev. Lett. 86, 5051 (2001).

${ }^{5}$ K. Avinash, S. V. Bulanov, T. Esirkepov, P. Kaw, F. Pegoraro, P. V. Sasorov, and A. Sen, Phys. Plasmas 5, 2849 (1998).

${ }^{6}$ M. B. Isichenko and Ya. L. Kalda, Sov. Phys. JETP 72, 1 (1991).

${ }^{7}$ R. L. Stenzel, M. C. Griskey, J. M. Urrutia, and K. D. Strohmaier, Phys. Plasmas 16, 2810 (2003).

${ }^{8}$ A. Fruchtman and Y. Maron, Phys. Fluids B 3, 1908 (1991).
}

${ }^{9}$ S. V. Basova, S. A. Varentsova, A. V. Gordeev, A. V. Gulin, and V. Yu. Shuvaev, Sov. J. Plasma Phys. 17, 2449 (1991).

${ }^{10}$ S. V. Bulanov, F. Pegoraro, and A. S. Sakharov, Phys. Fluids B 4, 2499 (1992).

${ }^{11}$ V. P. Ruban, Phys. Rev. E 65, 047401 (2002).

${ }^{12}$ D. Biskamp, E. Schwarz, and J. F. Drake, Phys. Plasmas 4, 1002 (1997).

${ }^{13}$ G. A. Askar'yan, S. V. Bulanov, F. Pegoraro, and A. M. Pukhov, Comments Plasma Phys. Controlled Fusion 17, 35 (1995); F. Califano, N. Attico, F. Pegoraro, G. Bertin, and S. V. Bulanov, Phys. Rev. Lett. 86, 5293 (2001).

${ }^{14}$ A. Runov, R. Nakamura, W. Baumjohann, R. A. Treumann, T. L. Zhang, M. Volwerk, Z. Vörös, A. Balogh, K. H. Glaßmeier, B. Klecker, H. Rème, and L. Kistler, Geophys. Res. Lett. 30, 11 (2003); T. Nagai, I. Shinohara, M. Fujimoto, S. Machida, R. Nakamura, Y. Saito, and T. Mukai, ibid. 108, A110, 357 (2003).

${ }^{15}$ B. Coppi, Phys. Lett. 11, 3 (1964); 12, 3 (1964).

${ }^{16}$ B. Coppi, G. Laval, and L. Pellat, Phys. Rev. Lett. 16, 1207 (1966); V. M. Vasyliunas, Rev. Geophys. Space Phys. 13, 303, and references therein.

${ }^{17}$ J. Wesson, Nucl. Fusion 30, 2545 (1990).

${ }^{18}$ M. Ottaviani and F. Porcelli, Phys. Rev. Lett. 71, 3802 (1993).

${ }^{19}$ N. M. Ferraro and B. N. Rogers, Phys. Plasmas 11, 4382 (2004).

${ }^{20}$ J. D. Huba, Phys. Rev. Lett. 72, 2033 (1994).

${ }^{21}$ J. F. Drake, R. G. Kleva, and M. E. Mandt, Phys. Rev. Lett. 73, 1251 (1994); D. Biskamp, E. Schwarz, and J. F. Drake, ibid. 76, 1264 (1996).

${ }^{22}$ M. B. Isichenko and A. M. Marnachev, Sov. Phys. JETP 66, 4, 702 (1987).

${ }^{23}$ S. V. Bulanov, M. Lontano, T. Zh. Esirkepov, F. Pegoraro, and A. M. Pukhov, Phys. Rev. Lett. 76, 3562 (1996); S. V. Bulanov, T. Zh. Esirkepov, M. Lontano, and F. Pegoraro, Plasma Phys. Rep. 23, 8, 660 (1997). ${ }^{24}$ V. V. Yan'kov, Phys. Plasmas 4, 571 (1997).

${ }^{25}$ D. A. Knoll and L. Chacòn, Phys. Rev. Lett. 88, 215003 (2002).

${ }^{26}$ T. K. M. Nakamura, D. Hayashi, M. Fujimoto, and I. Shinohara, Phys. Rev. Lett. 92, 5001 (2004).

${ }^{27}$ R. B. Dahlburg, P. Boncinelli, and G. Einaudi, Phys. Plasmas 4, 1213 (1997); 5, 79 (1998).

${ }^{28}$ G. Lapenta and D. A. Knoll, Sol. Phys. 214, 107 (2003).

${ }^{29}$ A. Frank, T. W. Jones, D. Ryu, and J. B. Gaalaas, Astrophys. J. 460, 777 (1996); D. Ryu, T. W. Jones, and A. Frank, ibid. 545, 475 (2000).

${ }^{30}$ G. Einaudi, Plasma Phys. Controlled Fusion 41, A293 (1999); A. F. Rappazzo, M. Velli, G. Einaudi, and R. B. Dahlburg, in Solar Wind 10, edited by M. Velli, R. Bruno, and F. Malara, AIP Conf. Proc. No. 679 (AIP, New York, 2003), p. 371.

${ }^{31}$ K. Nykyri and A. Otto, Geophys. Res. Lett. 28, 18 (2001).

${ }^{32}$ L. Chacón, D. A. Knoll, and J. M. Finn, Phys. Lett. A 308, 187 (2003).

${ }^{33}$ D. L. Jassby, Phys. Fluids 15, 1590 (1972).

${ }^{34}$ B. N. Kuvshinov, F. Pegoraro, and T. J. Schep, Phys. Lett. A 191, 296 (1994); T. J. Schep, F. Pegoraro, and B. N. Kuvshinov, Phys. Plasmas 1, 2843 (1994); B. N. Kuvshinov, V. P. Lakhin, F. Pegoraro, and T. J. Schep, J. Plasma Phys. 59, 727 (1998).

${ }^{35}$ M. Lesieur, Turbulence in Fluids: Stochastic and Numerical Modeling (Kluwer Academic, Dordrecht, 1990), pp. 64-74.

${ }^{36}$ B. N. Kuvshinov, E. Westerhof, T. J. Schep, and M. Berning, Phys. Lett. A 241, 287 (1998).

${ }^{37}$ N. Attico, F. Califano, and F. Pegoraro, Phys. Plasmas 8, 16 (2001).

${ }^{38}$ H. Baty, R. Keppens, and P. Comte, Phys. Plasmas 10, 12 (2003).

${ }^{39}$ N. Attico, F. Califano, and F. Pegoraro, Phys. Plasmas 7, 2381 (2000).

${ }^{40}$ A. Hasegawa and K. Mima, Phys. Rev. Lett. 39, 205 (1977); A. Hasegawa and K. Mima, Phys. Fluids 21, 115 (1978).

${ }^{41}$ B. Mandelbrot, in Penser Les Mathèmatiques, editions du Seuil, Paris, 1982, p. 226.

${ }^{42}$ P. G. Saffman, Vortex Dynamics (Cambridge University Press, Cambridge, 1992), p. 107.

${ }^{43}$ J. Bergmans and T. J. Schep, Phys. Rev. Lett. 87, 19 (2001).

${ }^{44}$ T. M. Abdalla, V. P. Lakhin, T. J. Schep, and E. Westerhof, Phys. Plasmas 10, 8 (2003).

${ }^{45}$ H. L. Berk, B. N. Breizman, and M. Pekker, Phys. Rev. Lett. 76, 1256 (1996). 